\title{
Expectativa de vida saudável para idosos brasileiros, 2003
}

\author{
Healthy life expectancy to Brazilian elders, 2003
}

M irela Castro Santos Camargos ${ }^{1}$

Roberto do Nascimento Rodrigues ${ }^{1}$

Carla Jorge M achado ${ }^{1}$

${ }^{1}$ Departamento de Demografia, Centro de

Desenvolvimento e

Planejamento Regional,

UniversidadeFederal de

M inas Gerais. Rua Curitiba

832/9o andar, Centro.

30170-120 Belo

HorizonteM G.

mirela@cedeplar.ufmg.br
Abstract The increase of the percentage of elderly population in Brazil and the increase in longevity incite a demand for information on the quantity of years spent in good health. The aim of the present study is to measure the life expectancy for the elderly of 60 years and above, by sex and age, in the year of 2003. The Sullivan method was used, which combined the life table with the current experience of mortality and the self-perceived health. The mortality information was obtained from the life tables published by the IBGE (Brazilian Institute of Geography and Statistics), 2003. Theself-perceived health was used and it was dichotomized in good and bad. This information came from the National Research of Household Sample (PNAD), 2003. The results indicate that women live longer, but spend a higher number of years perceiving their health as bad, as compared to men. The results also highlights to the need of considering the differences between sexes in relation to the demand for health care. It is also important to consider the need to have policies designed to allow the increase in the number of years that the elderly can live in good health conditions. Key words Health life expectancy, Elderly, Self-assessed health, PN AD 2003
Resumo No Brasil, o aumento da população idosa em relação à população total e 0 aumento da longevidade provocam uma demanda por informações sobre a quantidade de anos vividos com saúde. 0 objetivo do presente estudo é medir a expectativa de vida saudável para a população brasileira de 60 anos e mais, por sexo eidade, em 2003. Para isso, foi empregado o método de Sullivan, combinando a tábua de vida, com experiência de mortalidade corrente da população e suas percepções de saúde. As informações de mortalidadeforam obtidas de tábuas de vida publicadas pelo Instituto Brasileiro de Geografia e Estatística, 2003. O ptou-se por utilizar a autopercepção do estado de saúde, dicotomizada em boa e ruim, como medida do estado saúde dos indivíduos idosos, com informaçõesadvindas da Pesquisa $\mathrm{N}$ acional por Amostra Domiciliar (PNAD) de 2003. As estimativas mostram que as mulheres vivem mais, porém o número de anos a serem vividos por elas percebendo sua saúde como ruim é maior do que a estimativa para os idosos do sexo masculino. Os re sultados chamam atenção para a necessidade de considerar as diferenças entre os sexos em relação à demanda por cuidados de saúde, assim como para a necessidade de políticas visando aumentar os anos a serem vividos pelos idosos em condiçõesque estes considerem como de boa saúde.

Palavras-chave Expectativa de vida saudável, I dosos, Autopercepção de saúde, PNAD 2003 
Introdução

No Brasil, a população idosa aumenta em relação à população total e as pessoas passam a viver mais. N este contexto, cresceo interesse em investigar sea totalidade do número adicional de anos acrescido à expectativa de vida é vivida em condições de saúde adequada. 0 número de anos vividos com ou sem saúde, assim como o tipo de problemas de saúde experimentados por indivíduos idosos, exerce um papel fundamental no uso de serviços de saúde. Entretanto, o que se observa é que os dados usuais de mortalidade são insuficientes para mensurar as necessidades dos serviços. Afinal, é preciso saber não apenas o total de anos vividos, mas também 0 status de saúde da população a cada idade 1 .

Estimativas de expectativa de vida saudável têm sido utilizadas para suprir a demanda por informações sobre a quantidade de anos vividos com saúde. A esperança de vida saudável apresenta uma noção similar à expectativa de vida total, mas referese ao número médio de anos de vida que uma pessoa de determinada idade pode esperar viver com saúde, dado que prevaleçam as taxas de morbidadee mortalidadenaquela idadeespecífica. Sendo assim, a expectativa de vida total é composta pela quantidade de anos vividos, desde o nascimento ou a partir de uma determinada idade, em diferentes estados de saúde, atéa morte, sendo que os anos vividos com saúde fornecem a expectativa de vida saudável ${ }^{2}$. A vantagem da expectativa de vida saudável é que ela combina informações de morbidadee mortalidadeem um único índice, sendo de fácil interpretação ${ }^{3}$.

0 conceito de expectativa de vida saudável, assim como de indicadores de saúde, foi proposto pela primeira vez na década de sessenta ${ }^{4}$ e desenvolvido nos anos setenta 5 . Entreas formas demensuração, encontram-se o método da tábua de vida de duplo decremento, o método multiestado da tábua de vida e o método da prevalência observada na tábua de vida, ou método de Sullivan ${ }^{6,7}$. Para empregar os dois primeiros, são necessários dados longitudinais, que permitem mensurar a incidência. 0 método de Sullivan é o mais utilizado, pois usa dados correntes de prevalência de incapacidade, frequentemente obtidos em pesquisas ${ }^{1,2,6,7}$. No Brasil, nos primeiros anos da década de 2000, alguns estudos calcularam a expectativa de vida saudável, com base em dados de período e empregando o método de Sullivan, utilizando indicadores distintos de saúde $e^{8-14}$.

Assim como existem várias possi bilidades para se definir saúde, há diferentes maneiras de semensurar a expectativa de vida saudável como, por exemplo, expectativa de vida com e sem doenças, com e sem deficiências, com e sem incapacidade funcional, com percepção de saúde boa ou ruim ${ }^{6}$. Sendo assim, a forma com a qual o estado de saúde é operacionalizado depende dos objetivos do pesquisador e dos dados disponíveis.

A autopercepção de saúde tem sido muito utilizada em pesquisas quevisam estabelecer o estado de saúde do indivíduo, especialmente do idoso ${ }^{15}$ ${ }^{18}$. A pesar da sua natureza subjetiva, estudos têm demonstrado que a variável percepção de saúde apresenta boa confiabilidade e é uma medida válida ${ }^{19,20}$. A principal vantagem dessa variável éque 0 resultado da pergunta associa-se fortemente ao estado real ou objetivo de saúde das pessoas, sugerindo que a autopercepção da saúde pode ser utilizada como uma proxy das avaliações objetivas de saúde ${ }^{21}$. Além disso, por ser considerada um forte preditor de saúde e mortalidade ${ }^{22,23}$, a autopercepção de saúde tem sido utilizada para estimar a expectativa de vida saudável ${ }^{24}$.

Um estudo recente ${ }^{13}$, utilizando dados oriundos da Pesquisa M undial de Saúde(PM S), estimou a expectativa de vida saudável no Brasil com base em diferentes medidas de saúde, entre el as a autopercepção de saúde. Contudo, há ainda poucos estudos que estimaram expectativa de vida saudável para o Brasil, o que pode ser atribuído, em parte, à escassez de informações adequadas, como a autopercepção de saúde. A coleta dessa informação na PNAD 2003 oferece a oportunidade deanalisar a esperança de vida saudável para este ano. Uma vertente importante desta possibilidade reside na estimativa específica para a população idosa, posto que pode se constituir em instrumento valioso para o estabelecimento de políticas públicas voltadas para este segmento populacional.

0 objetivo do presenteestudo émedir a expectativa de vida saudável para os idosos brasileiros, por sexo e idade, para o ano de 2003. Tendo em vista as vantagens da utilização da autopercepção de saúde, neste trabalho, optou-se por empregá-la como medida do estado saúde dos indivíduos idosos.

\section{M etodologia}

\section{Dados}

As informações de mortalidade foram obtidas detábuas devida publicadas pelo Instituto Brasileiro de Geografia e Estatística (IBGE) ${ }^{25}$ para 0 ano de 2003. As prevalências de autopercepção de saúde, dicotomizadas em boa e ruim, foram estimadas com informações advindas da Pesquisa Nacional 
por Amostra Domiciliar (PNAD) ${ }^{26}$ de 2003, que contém um módulo especial desaúde. A PNAD cobretodo o país, com exceção da área rural de Rondônia, Acre, Amazonas, Roraima, Pará e Amapá.

$\mathrm{Na}$ PN AD analisada, a percepção do estado de saúde é avaliada por meio do quesito "estado de saúde segundo o próprio ponto de vista da pessoa, ou do responsável, no caso de criança pequena", com as seguintes opções de resposta: muito bom, bom, regular, ruim e muito ruim. N este estudo, a medida foi dicotomizada pela combinação das categorias "muito bom" e "bom", para fazer referência a uma boa autopercepção de saúde, e em "regular", "ruim" e "muito ruim", para referir uma autopercepção de saúde ruim. Essa mesma categorização foi utilizada por Romero ${ }^{27}$ ao trabalhar com a PNAD de 1998.

Como a percepção do estado de saúde envolve aspectos subjetivos, é de se esperar que haja diferença entre a percepção baseada nas respostas do próprio indivíduo e aquelas relatadas pelo informante. Estudos anteriores colocam que, em geral, o informante tende a classificar o estado de saúde em categorias piores do que aquel es cuja avaliação foi realizada pela própria pessoa ${ }^{15,28}$. Entretanto, optou-se por manter as informações dadas pelo informante para que fosse incluído um número maior de casos.

Para compor a amostra, foram incluídas as pessoas de 60 anos e mais. Foram excluídos os sujeitos sem declaração da idade (72) e do estado de saúde (2) e os casos de missing para o estado de saúde (2), sendo a amostra composta por 35.038 idosos.

Asinformações de prevalências de autopercepção de saúde boa e ruim foram processadas no programa Statistical Package for the Social Sciences (SPSS), versão 13.0. Foram utilizadosos pesos existentes na própria base, para garantir a representatividade da população total.

\section{M étodo}

Para estimar a expectativa de vida saudável, foi utilizado o método introduzido por Sullivan ${ }^{5}$, combinando a tábua de vida, com experiência de mortalidade corrente da população em 2003, e as prevalências de saúde na população no mesmo período. Assim, o número de anos a serem vividos em condições de saúde boa ou ruim é estimado aplicando a prevalência deautopercepção de saúdeboa e ruim aos anos-pessoas derivados da tábua de vida de período.
A principal vantagem da utilização do método deSullivan para monitorar ten dências deexpectativa de vida saudável é que são necessários apenas dados transversais. Por outro lado, ao empregar dados de período, não estão incorporadas possíveis mudanças em relação a melhoras nas condições de saúde e nas taxas de mortalidade da população. Ademais, o método não permite avaliar transições de uma condição de saúde para outra, como por exemplo, o retorno de um indivíduo que avalia sua saúde como ruim para boa, o que só é analisado no modelo multiestado, com uso de dados Iongitudinais deincidência. No entanto, estudos prévios têm demonstrado que, caso não existam alterações repentinas tanto nas prevalências como nas taxas de mortalidade, o método de Sullivan é bastante confiável para este tipo de análise ${ }^{29}$. Sendo assim, acredita-sequeas estimativas realizadas aqui se ajustam à população idosa brasileira de 2003.

As tabelas de sobrevivência foram construídas separadamente para homens e mulheres. 0 número de anos vividos dentro de cada idade na tábua de vida foi distribuído segundo a prevalência em cada grupo etário específico. Assim, o total de anos vividos com autopercepção de saúde boa e ruim permitiu calcular a expectativa de vida com autopercepção de saúde boa e expectativa de vida com autopercepção de saúde ruim. Ou seja:

$$
\begin{aligned}
& \operatorname{EVSB}_{x}=\frac{\sum\left(1-{ }_{n} \pi_{x}\right)_{n} L_{x}}{l_{x}} \\
& \operatorname{EVSR}_{x}=\frac{\sum\left({ }_{n} \pi_{x}\right)_{n} L_{x}}{l_{x}}
\end{aligned}
$$

Sendo que:

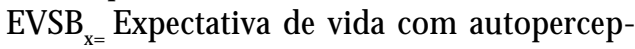
ção de saúde boa. Corresponde ao número médio de anos a serem vividos com autopercepção de saúde boa a partir da idade $x$.

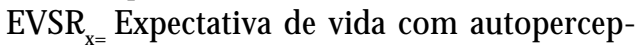
ção de saúde ruim. Corresponde ao número mé dio de anos a serem vividos com autopercepção de saúde ruim a partir da idade $x$.

$\Sigma\left[1-{ }_{n} \pi_{x}{ }_{n}{ }_{n} L_{x}:\right.$ Total de anos vividos com autopercepção de saúde boa desde a idade $x$. É obtido somando todos os $\left[1-{ }_{n} \pi_{x}\right]{ }_{n} L_{x}$ desde a idade $x$ até 0 grupo etário final $(85+)$.

$\Sigma\left[{ }_{n} \pi_{\mathrm{x}}\right]_{{ }_{n}}^{*} L_{\mathrm{x}}$ : Total de anos vividos com autopercepção de saúde ruim desde a idade $x$.

$I_{x}$ : Probabilidade de sobreviver até a idade $x$. 
Resultados

$\mathrm{Na}$ Tabela 1, estão apresentadas as estimativas de expectativa de vida total e as expectativas de vida com autopercepção de saúde boa e ruim, em termos absolutos e relativos, para 2003, por sexo e idade. Para facilitar a análise, foram selecionadas as idades 60,70 e 80 anos. Além disso, foram construídas razões entre mulheres e homens.

As estimativas mostram que as mulheres idosas apresentam uma vantagem em relação aos homens no que diz respeito à expectativa de vida em todas as idades. Aos 60 anos, por exemplo, as mulheres podem esperar viver um total de anos adicionais que é cerca de $15 \%$ superior ao total de anos a serem vividos pelos homens.

Em relação às condições de saúde nos anos a serem vividos, em 2003, o que se observa é que as mulheres apresentam maiores expectativas de vida com autopercepção de saúde boa mas, em termos proporcionais, a vantagem passa a ser masculina. Além disso, as expectativas de vida com autopercepção de saúde ruim são maiores entre as mulheres idosas, em termos absolutos e relativos. Portanto, a vantagem feminina em relação ao número total de anos a serem vividos torna-se uma desvantagem quando analisadas as condições de saúde nos anos de vida remanescentes.

Outro fato que chama atenção é que o número de anos a serem vividos percebendo a saúde como ruim será maior, em ambos os sexos e em todas as idades analisadas, se comparados aos anos vividos com percepção de saúde boa.

\section{Discussão}

Um dos principais fatores explicativos para as diferenças na expectativa de vida por idade éa mortalidadediferencial entrehomensemulheres ${ }^{30}$. Deacordo com o presente estudo, em 2003, as mulheres idosas apresentam não apenas maiores expectativas de vida como também podem esperar viver um número maior deanospercebendo sua saúde como boa. Entretanto, se comparadas aos homens, as mulheres apresentam uma maior proporção de anos a serem vividos percebendo sua saúde como ruim.

A desvantagem das mulheres na proporção de anos a serem vividos com piores condições de saúde tem sido mostrada em vários trabalhos ${ }^{8-12,22,29,30}$ e tem sido motivo de discussão. Acredita-se que a maior sobrevivência feminina, permitindo que elas atinjam idades mais avançadas, nas quais o declínio da capacidade funcional é mais evidente, seja um dos principais fatores explicativos para essa diferença entre os sexos. Além disso, existeo argumento de que as condições de saúde das mulheres idosas podem ser reflexo de condições econômicas, sociais e culturais desiguais, nos diversos momentos de suas vidas ${ }^{33,34}$. Não obstante, este argumento deveser aceito com cautela, uma vez queimplicaria uma esperança de vida maior para os homens, a cada idade, ao longo de quase todo o ciclo de vida, o que não acontece nas populações humanas.

0 que parece razoável considerar, também, é que taxas de mortalidade mais elevadas dos homens em idades mais jovens poderiam permitir

Tabela 1. Estimativas de expectativa de vida total e com autopercepção de saúde boa e ruim, por idade e sexo. Brasil, 2003.

\begin{tabular}{|c|c|c|c|c|c|c|}
\hline Sexo & $\begin{array}{l}\text { Idade } \\
\text { exata }\end{array}$ & $\begin{array}{c}\text { Expectativa } \\
\text { de vida } \\
\text { total }\end{array}$ & $\begin{array}{l}\text { Expectativa de } \\
\text { vida com } \\
\text { autopercepção } \\
\text { de saúde boa }\end{array}$ & $\begin{array}{l}\text { Expectativa de } \\
\text { vida com } \\
\text { autopercepção } \\
\text { de saúde ruim }\end{array}$ & $\begin{array}{l}\text { \% de anos a serem } \\
\text { vividos com } \\
\text { autopercepção de } \\
\text { saúde boa }\end{array}$ & $\begin{array}{l}\% \text { de anos a serem } \\
\text { vividos com } \\
\text { autopercepção de } \\
\text { saúde ruim }\end{array}$ \\
\hline \multirow[t]{3}{*}{ Homens } & 60 & 19,1 & 8,4 & 10,6 & 0,44 & 0,56 \\
\hline & 70 & 13,1 & 5,0 & 8,1 & 0,38 & 0,62 \\
\hline & 80 & 8,8 & 2,9 & 5,8 & 0,34 & 0,66 \\
\hline \multirow[t]{3}{*}{ Mulheres } & 60 & 22,1 & 8,9 & 13,1 & 0,41 & 0,59 \\
\hline & 70 & 15,0 & 5,7 & 9,4 & 0,38 & 0,62 \\
\hline & 80 & 9,6 & 3,4 & 6,2 & 0,36 & 0,64 \\
\hline Mulheres/ & 60 & 1,16 & 1,06 & 1,23 & 0,92 & 1,06 \\
\hline \multirow[t]{2}{*}{ Homens } & 70 & 1,15 & 1,14 & 1,16 & 0,99 & 1,00 \\
\hline & 80 & 1,10 & 1,17 & 1,06 & 1,07 & 0,97 \\
\hline
\end{tabular}

Fonte dos dados básicos: Instituto Brasileiro de Geografia e Estatística25,26. 
que, na velhice, a composição do grupo etário masculino fosse mais favorável que o feminino, devido a um mecanismo de seleção ${ }^{35}$. I sto resultaria num grupo heterogêneo, no qual haveria um número maior de idosas mais fragilizadas e susceptíveis do que idosos, o que seria refletido diretamente nos anos a serem vividos percebendo sua saúde como ruim.

M as há que se considerar também o enorme diferencial na percepção demorbidadee condições de saúde entre mulheres e homens. As mulheres declaram um volume maior de doença ou condições mórbidas do que os homens ${ }^{36}$, embora não necessariamente isto signifique dizer que a saúde delas seja pior. Além disso, deve-se ressaltar o fato de quea mulher, ao desenvolver práticas mais constantes de consulta médica relacionada à sua saúde sexual ereprodutiva, acabam por desenvolver percepção mais acurada sobre morbidade e condições de saúde do que os homens.

Ainda em relação ao uso da auto-avaliação do estado de saúde como indicador de saúde, é preciso considerar que, como se trata de autopercepção, al guns diferenciais podem guardar relação com diversosaspectos, tais como interpretação diferente do indivíduo daquilo que sente, de acordo com sua experiência, cultura, escolaridade e do contexto social em que está inserido. Ademais, como as informações são autoreferidas, as variáveis são susceptíveis a vários tipos de erros de medida ${ }^{15}$. Contudo, apesar de sua natureza subjetiva, a medida de autopercepção do estado de saúde tem sido amplamente utilizada em pesquisas que visam estabelecer o estado de saúde do indivíduo, especialmente do idoso, eque é uma das formas indicadas pela REVES, uma rede internacional de pesquisadores no assunto, para estimar a expectativa de saudável. 6 .

Um outro aspecto que deve ser considerado éa forma como se operacionaliza a variável autopercepção de saúde. Um exemplo disso pode ser observado quando se compara os resultados do presente estudo com outro que também estimou a expectativa de vida saudável para o Brasil em $2003^{13}$. Apesar dos dois estudos encontrarem o mesmo comportamento ao analisar as diferenças entre os sexos, eles classificam de forma diferente aqueles que declararam a saúde como "regular" ao dicotomizar a variável autopercepção de saúde. Ao optar incluí-los na categoria "boa", os autores encontraram menores valores na expectativa de vida com autopercepção de saúde ruim, se comparados aos do presente trabalho.

0 presente estudo mostrou que, com o avançar da idade, as diferenças entre os sexos diminu- em em relação à expectativa de vida eà proporção de anos a serem vividos percebendo a saúde como ruim. Este comportamento leva a crer que os "idosos mais idosos" seriam mais homogêneos em relações às condições saúde, ou seja, nas idades mais avançadas o efeito da seletividade seria menor.

Ainda em relação aos diferenciais entre os sexos, cabe ressaltar que, em termos absolutos, a maior longevidade feminina permite que as mulheres vivam um número maior de anos percebendo sua saúde como boa. Em se tratando de políticas públicas, essa vantagem feminina em termos absolutos deve ser vista como uma oportunidade para que elas tenham um tempo maior para se prevenir contra possíveis patologias, atenuando sua intensidade e postergando ao máximo o seu aparecimento.

Independente da discussão sobre as diferenças entre os sexos, os resultados encontrados neste estudo apontam para um desequilíbrio em relação aos anos a serem vividos percebendo a saúde como boa e ruim, ou seja, nas idades analisadas, nota-se que a proporção de anos vividos com autopercepção ruim é expressivamente maior. Este desequilíbrio pode ser atribuído à forma utilizada para categorizar a autopercepção de saúde; afinal, ao inserir aqueles que auto-avaliaram sua saúde como regular na categoria ruim, seu peso foi ampliado. Ao fazer esta escolha de categorização, pensou-se em quanto tempo os indivíduos viveriam em média percebendo sua saúde como muito boa ou boa e o que poderia ser feito para ampliar o número de anosnesta condição. Assim, em termos de políticas públicas, a questão que se coloca é como fazer com que os idosos vivam um número maior de anos percebendo sua saúde como boa e diminuam a expectativa de vida com autopercepção desaúderuim?

Tendo em vista que o envel hecimento saudável é consequência da interação de diversos fatores, as políticas públicas devem promover ações integradas que abordem, simultaneamente, os principais fatores que interferem diretamente na autopercepção desaúdedos idosos, de forma a contribuir para a promoção da saúde e do bem-estar e, consequentemente, para a qualidade de vida dos idosos.

Para isso, é preciso reconhecer que os problemas de saúde enfrentados pelos idosos não se iniciam quando se atinge 60 anos, mas são o resultado, dentre outros fatores, de um acúmulo de experiências passadas, dos cuidados com a saúde, das condições de moradia, educação, alimentação e higiene, da prática deatividadefísica e das oportunidades perdidas ou aproveitadas. Assim, é importante que as políticas sociais e de saúde estejam estabelecidas de forma a garantir a promoção da saúde durante todo o ciclo de vida do indivíduo. 
Finalmente, é preciso considerar que, em populações que estão envelhecendo, como é o caso do Brasil, as melhoras nas condições de saúde podem ajudar a reduzir gastos com cuidados com saúde e, ao mesmo tempo, minimizar a sobrecarga gerada no sistema de saúde em decorrência do envelhecimento populacional.

\section{Colaboradores}

M CS Camargos trabalhou na concepção teórica, elaboração, análise e redação final do texto e RN Rodrigues e CJ M achado participaram de todas as etapas de análise e na redação do texto.

\section{Agradecimentos}

Os autores agradecem ao CN Pq Brasil.

\section{Referências}

1. Portrait F, M aarten L, Degg D. Life expectancies in specific health states: results from a joint model of health status and mortality of older persons. Demography 2001; 38(4):525-536.

2. Jagger $\mathrm{C}$. H ealth expectancy calculation by the Sullivan Method: a practical guide [NUPRI Research Paper, n. 68]. Madison: NUPRI; 1999.

3. Valkonen T, Sihvonen A, Lahelma E. Health expectancy by level of education in Finland. Soc Sci M ed 1997; 44(6):801-808.

4. Sanders BS. M easuring community health levels. Am J Public Health 1964; 54(7):1063-1070.

5. Sullivan DF. A single index of mortality and morbidity. HSM HA Health Reports 1971; 86:347-354.

6. Robine J-M, Romieu I, Cambois E. Health expectancy indicators. Bull World Health Organ 1999; 77(3):181-185.

7. Manton KG, Land KC. Active life expectancy estimates for U.S. elderly population: a multidimensional continuous-mixture model of functional change applied to completed cohorts. Demography 2000; 37(3):253-265.

8. Instituto Brasileiro de Geografia e Estatística. Censo demográfico 2000: características gerais da população, resultados da amostra. Rio de Janeiro: IBGE, 2003.

9. Baptista DBDA. Idosos no município de São Paulo: expectativa de vida ativa e perfis multidimensionais de incapacidade a partir da SABE [dissertação]. Belo Horizonte (MG): Universidade Federal de M inas Gerais; 2003.

10. Camargos M CS. Estimativas de expectativa de vida livre de e com incapacidade funcional: uma aplicação do mé todo de Sullivan para idosos paulistanos, 2000 [dissertação]. Belo Horizonte (M G): U niversidade Federal de Minas Gerais; 2004.

11. Camargos MCS, Perpetuo IHO, Machado CJ. Expectativa de vida com incapacidade funcional em idosos em São Paulo, Brasil. Rev Panam Salud Publica/Pan Am. J. Public Health 2005; 17(5-6):379-386.

12. Camargos M CS, Machado CJ, Rodrigues, RN. Disability life expectancy for the elderly, city of São Paulo, Brazil, 2000: gender and educational differences. J Biosoc Sci 2007; 39(3):455-463.

13. Romero DE, Leite IC, Szwarcwald CL. Healthy life expectancy in Brazil: applying the Sullivan method. Cad Saude Publica 2005; 21(Supl. 1):S7-S18. 
14. Santos JLF. Análise de sobrevida sem incapacidades. In: Lebrão ML, Duarte YAO, organizadoras. 0 projeto SABE no Brasil: uma abordagem inicial. Brasília: Organização Pan-Americana da Saúde; 2003. p.167-182.

15. Alves LC. D eterminantes da autopercepção de saúde dos idosos do município de São Paulo, 1999/2000 [dissertação]. Belo Horizonte (MG): Universidade Federal de Minas Gerais; 2004.

16. Alves LC, Rodrigues RN. Determinantes da autopercepção de saúde entre idosos do município de São Paulo, Brasil. Rev Panam Salud Publica/Pan Am. J. Public Health 2005; 17(5-6):333-341.

17. Baron-Epel O, Kaplan G. General subjective health status or age-related subjective health status: does it make a difference? Soc Sci M ed 2001; 53(9):1373-1381.

18. Dachs JNW, Santos APR. Auto-avaliação do estado de saúde no Brasil:análise dos dados da PNAD/2003. Cien Saude Colet 2006; 11(4):887-894.

19. Martikainen P, Aromaa A, Heliövaara M, Klaukka T, Knekt P, M aatela J, Lahelma E. Reliability of perceived health by sex and age. Soc Sci M ed 1999; 48(8):1117-1122.

20. Bailis DS, Segall A, Chipperfield JG. Two views of selfrated general health status. Soc Sci M ed 2003; 56(2):203217.

21. Appels A, Bosma H, Grabauskas V, Gostautas A, Sturmans F. Self-rated health and mortality in a Lithuanian and a Dutch population. Soc Sci M ed 1996; 42(5):681689.

22. Kaplan G A, Camacho T. Perceived health and mortality: a nine-year follow-up of the human population laboratory cohort. Am J Epidemiol 1983; 117(3):292-304.

23. Idler EL, Benyamini Y. Self-rated health and mortality: a review of twenty-seven community studies. J Health Soc Behav 1997; 38(1):21-37.

24. Ofstedal MB, Zimmer Z, Cruz G, Chan A, Lin Y. Selfassessed health expectancy among older Asians: a comparison of Sullivan and multistate life table methods. [Report No. 03-60]. Ann Arbor: Population Studies Center, 2003.

25. Instituto Brasileiro de Geografia e Estatística. Tábuas Completas de M ortalidade - 2003. [acessado 2005 nov 25]. Disponível em: ftp://ftp.ibge.gov.br/Tabuas_ Completas_de_Mortalidade/Tabuas_Completas_de_ M ortalidade_2003
26. Instituto Brasileiro de Geografia e Estatística. Pesquisa Nacional por Amostra de Domicílios, 2003. Rio de Janeiro: IBGE; 2005.

27. Romero $D E$. Diferenciais de gênero no impacto do arranjo familiar no status de saúde dos idosos brasileiros. Cien Saude Colet 2002; 7(4):777-794.

28. Dach JN. Determinantes das desigualdades na autoavaliação do estado de saúde no Brasil: análise dos dados da PNAD/1998. Cien Saude Colet 2002; 7(4):641-657.

29. Mathers CD, Robine JM. How good is Sullivan's method for monitoring changes in population health expectancies? J Epidemiol Community Health 1997; 51(1):80-86.

30. Berquó E. Algumas considerações demográficas sobre o envelhecimento da população no Brasil. In: Anais do Seminário Internacional sobre Envelhecimento Populacional: U ma Agenda para o Fim do Século; 1996; Brasília. p.16-34.

31. Agree, EM. The influence of personal care and assistive devices on the measurement of disability. Soc Sci M ed 1999; 48(4):427-443.

32. Zimmer Z. Active life expectancy and functional limitations among older Cambodians: results from a 2004 survey. Working Papers No. 201]. New York: Population Council; 2005.

33. Barreto SM, Giatti L, U chôa E, Lima-Costa, MF. Gênero e desigualdades em saúde entre idosos brasileiros. In: Anais da O ficina de Trabalho sobre Desigualdades Sociais e de Gênero em Saúde de Idosos no Brasil; 2002; O uro Preto. p.59-69.

34. Goldani AM. Mulheres e envelhecimento: desafios para novos contratos intergeracionais e de gênero. In: Camarano AA, organizadora. Muito além dos 60: os novos idosos brasileiros. Rio de Janeiro: IPEA; 1999. p. 75-113.

35. Perls T, Kunkel LM, Puca AA. The genetics of exceptional human longevity. J Mol Neurosci. 2002; 19(12):233-238.

36. MCD onough $P$, Walters V. Gender and health: reassessing patterns and explanations. Soc Sci M ed 2001; 52(4):547-559.

Artigo apresentado em 02/03/2007

Aprovado em 16/10/2007

Versão final apresentada em 16/01/2007 